\title{
Pegfilgrastim in primary prophylaxis of febrile neutropenia following frontline bendamustine plus rituximab treatment in patients with indolent non-Hodgkin lymphoma: a single center, real-life experience
}

\author{
Claudio Cerchione $^{1}$ (D) Amalia De Renzo ${ }^{1} \cdot$ Maria Di Perna $^{1} \cdot$ Roberta Della Pepa $^{1}$. \\ Novella Pugliese ${ }^{1} \cdot$ Lucio Catalano $^{1} \cdot$ Fabrizio Pane $^{1} \cdot$ Marco Picardi $^{2}$
}

Received: 13 July 2016 / Accepted: 26 October 2016 /Published online: 3 November 2016

(C) The Author(s) 2016. This article is published with open access at Springerlink.com

\begin{abstract}
Background In this prospective study, the impact of granulocyte colony-stimulating factors (G-2 CSF) administered during induction treatment with bendamustine plus rituximab for indolent non- Hodgkin Llymphoma (NHL) was evaluated by comparing patients who received secondary prophylaxis with filgrastim (control group) versus. patients who received pegfilgrastim as primary prophylaxis (peg-group). The primary endpoint was the incidence rate of febrile neutropenia (FN)- related chemotherapy disruptions (regarding dosedense and/or dose-intensity of schedule). The Ssecondary endpoint included days of hospitalization due to FN, and GCSF-related side effects (grade $\geq 3$ WHO toxicity criteria) in each group.

Methods One hundred twenty-two: 122 consecutive patients, with untreated indolent NHL, were referred to our outpatient unit for remission induction immuno-chemotherapy with bendamustine-rituximab. During the first period, 61 patients received secondary prophylaxis with filgrastim, given "on demand" if ANC was $<1000 / \mathrm{mm} 3$. During the second period, 61 patients received primary prophylaxis with pegfilgrastim in a single administration.
\end{abstract}

Claudio Cerchione

claudiocerc@hotmail.com

1 Hematology, Department of Clinical Medicine and Surgery, Azienda Ospedaliera Universitaria Federico II, Via Pansini 5, 80131 Naples, Italy

2 Department of Advanced Biomedical Science, Azienda Ospedaliera Universitaria Federico II, Via Pansini 5, 80131 Naples, Italy
Results Pegfilgrastim was significantly associated with fewer incidence rate of FN-related chemotherapy disruptions (11.4\% in the control group vs. $1.6 \%$ in the peg-group, $p=0.04$ ) and fewer days of hospitalization due to FN (median number 18 days in the control group vs. 6 in the peg-group, $p=0.04$ ). In terms of G-CSF-related extra-hematological grade III side effects, no significant difference has been found in the two groups $(9.8 \%$ in the control group vs. $11.5 \%$ in the peg-group, $\mathrm{p}=0.77$ ). Only one patient stopped the treatment in the peggroup due to intolerance.

Conclusions In patients with indolent NHL, in front-line treatment with bendamustine plus rituximab, primary prophylaxis with pegfilgrastim seems to reduce the incidence of chemotherapy disruptions due to $\mathrm{FN}$, and the days of hospitalization. Moreover, it is well- tolerated and may increase the opportunity to maintain the planned schedule of treatment. These results make pegfilgrastim an advantageous option in most cases both in terms of cost-effectiveness and quality of life. These preliminary observations need to be validated by controlled clinical trials.

\section{Introduction}

Bendamustine is a bifunction alkylating agent that produces both single- and double-strand breaks in deoxyribonucleic acid. It is frequently used in association with rituximab as a frontline treatment of indolent (follicular and non-follicular) non-Hodgkin lymphoma (NHL). With this schedule, myelotoxicity, in particular severe neutropenia, is the main expected side effect $[1,2]$. Febrile neutropenia $(\mathrm{FN})$ is one of the most important clinical signs of infection during chemotherapy and is characterized by an absolute neutrophil count (ANC) $<1000 / \mathrm{mm}^{3}$ and at least one temperature 
measuring of $\geq 38^{\circ} \mathrm{C}$ [3]. Once an episode of FN occurs, the risk of FN increases in subsequent chemotherapy courses [4-6]. FN may lead to a disruption of chemotherapy program, with delay of cytotoxic agent administration and/or reduction of relative dose intensity, adversely affecting long-term patients' outcome [4-6]. The American Society of Clinical Oncology and the National Comprehensive Cancer Network recommend the use of granulocyte colony-stimulating factors (G-CSF), which have been developed to stimulate the proliferation and differentiation of neutrophils in patients receiving cytotoxic agent treatments [7, 8]. The routine use of G-CSF from the first cycle of myelosuppressive chemotherapy, i.e., primary prophylaxis, is indicated when the overall FN risk is greater than $20 \%[6,7]$. In the other cases ( $\leq 20 \%$ of risk), it is suggested a secondary prophylaxis, which consists of postchemotherapy G-CSF administration "on demand" if ANC is $<1000 / \mathrm{mm}^{3}[6,7]$. Another important issue is the type of G-CSF to employ [9-11]. Filgrastim is a non-pegylated form of G-CSF, used at the daily dose of $5 \mu \mathrm{g} / \mathrm{kg}$, until the end of neutropenia, according to the myelosuppressive chemotherapy schedules [7]. Pegfilgrastim is a pegylated long-acting recombinant form of G-CSF which extends the half-life, requiring less frequent administrations than non-pegylated G-CSF [9]. Pegfilgrastim is cleared via a neutrophil-mediated system and requires only a single dose administered once per chemotherapy cycle [9-11]. It is indicated to decrease the incidence of infections in patients with non-myeloid malignancies, receiving myelosuppressive chemotherapy [6]. However, data on the optimal G-CSF strategy, i.e., primary vs. secondary prophylaxis and/or non-pegylated vs. pegylated form, are scanty in the setting of patients with NHL undergoing frontline treatment with immuno-chemotherapy schedule $[1,2]$.

In this prospective study, the impact of G-CSF administered during induction treatment with bendamustine plus rituximab for indolent NHL was evaluated by comparing patients who received secondary prophylaxis with filgrastim (control group) to patients who received pegfilgrastim as primary prophylaxis (peg-group). The primary endpoint was the incidence rate of FN-related chemotherapy disruptions (regarding dose dense and/or dose intensity of schedule). Secondary endpoints included days of hospitalization due to FN and G-CSF-related side effects (grade $\geq 3$ WHO toxicity criteria) in each group.

\section{Patients and methods}

\section{Study design}

From March 2013 to February 2016, 264 patients with histologically diagnosed and untreated indolent NHL (including grade 1 or 2 follicular lymphoma, lymphoplasmocitic lymphoma, small lymphocytic lymphoma, and marginal zone lymphoma), age $\geq 18$ years, and WHO performance score 0 2 were screened for enrollment. Eligible criteria were features requiring to start immuno-chemotherapy treatment, i.e., Ann Arbor stage III or IV, and impaired hemopoiesis (hemoglobin $<10 \mathrm{~g} / \mathrm{dL}, \mathrm{ANC}<1500 / \mathrm{mm}^{3}$, or platelet count $<100 \times 10^{9} / \mathrm{L}$ ), presence of B symptoms, large tumor burden (three areas $>5 \mathrm{~cm}$, or one area $>7.5 \mathrm{~cm}$ ), bulky disease with impingement on internal organs, progressive disease (defined as a more than $50 \%$ increase of tumor mass within 6 months), and/or a hyperviscosity syndrome [12]. Only patients who received bendamustine plus rituximab regimen were included in the study (Fig. 1).

Ninety-nine patients were excluded: 79 because they underwent to other chemo-immunotherapy regimens, while 20 because of severe cardiac disease or previous malignancy, inadequate hepatic, renal, or cardiac function, or infection with HIV or hepatitis B (HbsAg positivity) (Fig. 1).

All patients underwent standard pretreatment screening, including a physical examination, complete blood count, assessment of serum chemistry, serum immune-electrophoresis, measurement of immunoglobulin concentrations, chest radiograph, CT scans of the chest, abdomen, and pelvis, sonography of the abdomen, and bone marrow aspiration and biopsy.

The protocol was approved by the local ethics committee; the study complied with the Declaration of Helsinki and its amendments. It was done in accordance with the Good Clinical Practice guidelines. All patients gave written informed consent.

\section{Strategies of prophylaxis with G-CSF}

All patients received frontline immune-chemotherapy courses in our outpatient unit. Treatment included intravenous bendamustine $\left(90 \mathrm{mg} / \mathrm{m}^{2}\right.$ given over $30-60 \mathrm{~min}$ on days 1 and 2 of each cycle) plus rituximab $\left(375 \mathrm{mg} / \mathrm{m}^{2}\right.$ on day 1 of each cycle), every 4 weeks for up to 6 cycles [1].

Patients were divided into two groups of G-CSF prophylactic strategy. From March 2013 to August 2014 (first period), 61 patients received secondary prophylaxis with filgrastim $(5 \mu \mathrm{g} / \mathrm{kg} /$ day s.c. for at least 3 days $)$ given on demand if ANC was $<1000 / \mathrm{mm}^{3}$. Since July 2014 (second period), the use of primary prophylaxis with pegfilgrastim (Neulasta ${ }^{\circledR}$; Amgen) became in our division a standard practice during frontline treatment for NHL with bendamustine plus rituximab. Thus, during the second period, 61 patients received primary prophylaxis with pegfilgrastim $(6 \mathrm{mg}$, injected subcutaneously in a single administration on day 4 , from the first course of immuno-chemotherapy).

All neutropenic patients underwent antimicrobial prophylaxis with quinolones and azoles, as elsewhere reported [13]. 


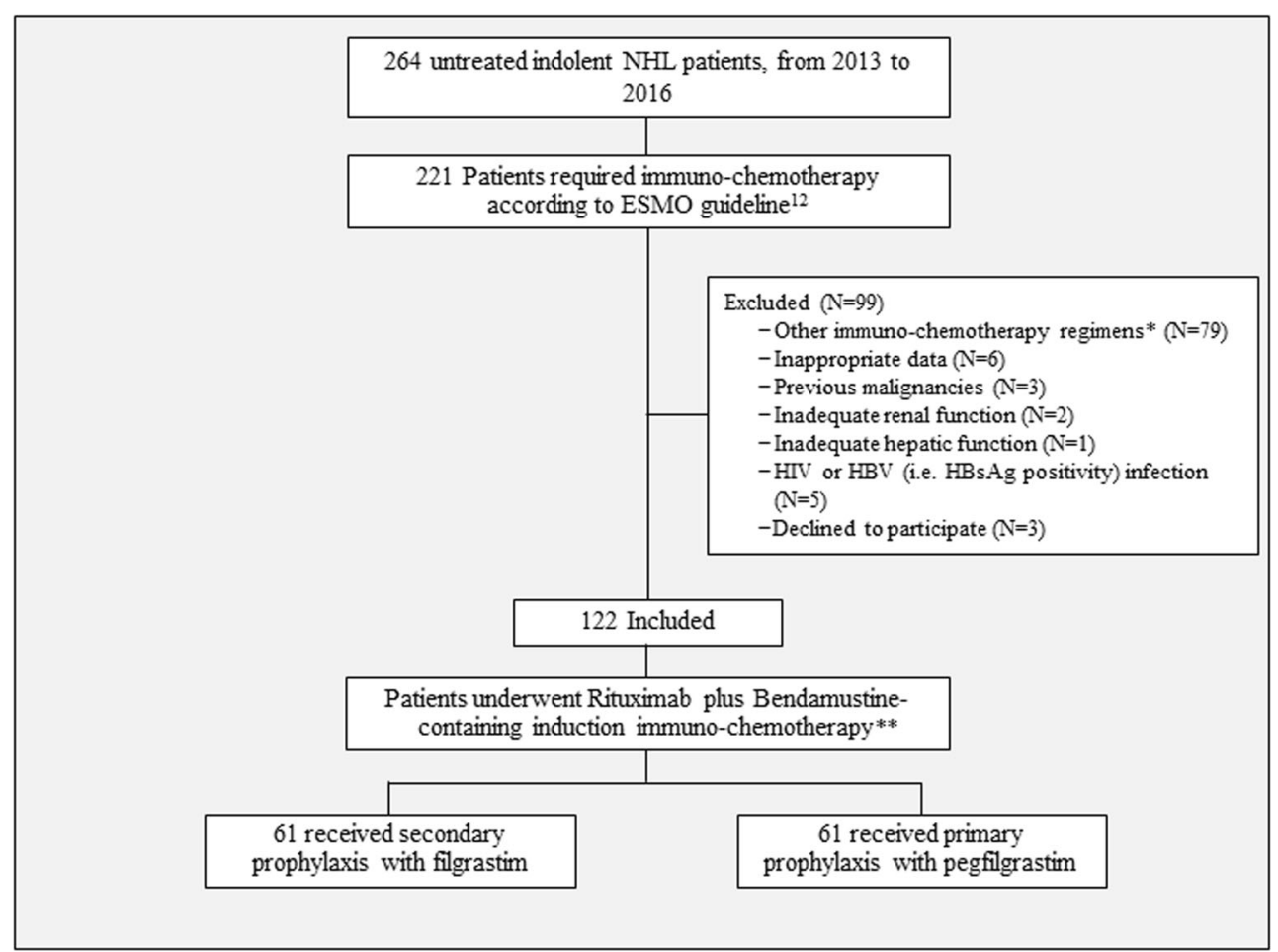

Fig. 1 Flow chart shows patient selection during the study. *Other immuno-chemotherapy regimens included R-CHOP, R-CVP, and R-FC [15]. **Immuno-chemotherapy with rituximab plus bendamustine was conducted according to Rummel et al. study [1]

\section{Study endpoints}

We defined as "disrupted chemotherapy" when we delayed treatment cycles for at least 1 week if the leukocyte count was less than $2000 / \mathrm{mm}^{3}$ before a scheduled cycle ("time-disruption"), or if we noted a leukocyte count less than $1000 / \mathrm{mm}^{3}$ on two consecutive days between cycles, the dose of bendamustine was decreased to $70 \mathrm{mg} / \mathrm{m}^{2}$ ("dose-disruption") [1].

During the study period, as part of our Institutional guidelines for post-chemotherapy supportive care, in patients with $\mathrm{FN}$, i.e., an $\mathrm{ANC}<1000 / \mathrm{mm}^{3}$ and at least one temperature measuring of $\geq 38{ }^{\circ} \mathrm{C}$, blood cultures were performed every 24-48 h: the Vitek 2 automated system (bioMérieux, Marcy l'Etoile, France) was used for bloodstream isolate identification and antimicrobial susceptibility testing. Minimum inhibitory concentrations (MICs) were evaluated by using E-test (BioMerieux) strips and classified according to the European Committee on Antimicrobial Susceptibility Testing [14]. If respiratory symptoms or signs appeared, sputum culture and chest radiography or thorax computed tomography (CT) scans were performed. Serum CMV DNA screening was performed in case of neutropenic fever or in patients with fever even in the absence of neutropenia.

We used WHO's toxicity criteria to assess treatmentrelated toxic effects [3]. Complete blood counts, including differential counts, and physical examination were done twice a week.

\section{Statistical analysis}

For the statistical evaluations, the $\chi^{2}$ test was performed to compare proportions for clinical characteristics and complication rate, and the $t$ test was used to compare the quantitative variables of clinical characteristics, disruptions, and hospitalization times between the two groups. $p$ values less than 0.05 were considered to indicate a significant difference.

\section{Results}

During the study period, among 264 patients with diagnosis of indolent NHL, 122 consecutive patients (63 males and 59 females), older than 18 years (median age, 45.3 years; range, $31-$ 77) with untreated indolent NHL (histological subtypes: follicular, $n=83$; marginal zone, $n=32$; small lymphocytic, $n=4$; and lymphoplasmocytic, $n=3$ ), met the inclusion criteria and were included in the final analysis (Table 1). All patients underwent to first-line immuno-chemotherapy with bendamustine plus rituximab.

\section{Findings in the control group}

In the 366 cycles of immuno-chemotherapy performed in the control group, the median number of the vials of filgrastim administered was 3 (range, $0-5$ ), started in mean from the second course (range, 1-3). The mean of nadir of ANC was 
Table 1 Baseline characteristics of patients in the two study groups

\begin{tabular}{|c|c|c|c|}
\hline & Control group & Peg-group & $\begin{array}{l}p \\
\text { value }\end{array}$ \\
\hline Total patients & 61 & 61 & \\
\hline \multicolumn{4}{|l|}{ Sex } \\
\hline Male & $32(52.4)$ & $31(50.8)$ & \multirow[t]{2}{*}{0.85} \\
\hline Female & $29(47.6)$ & $30(49.2)$ & \\
\hline \multicolumn{4}{|l|}{ Age, years } \\
\hline Median (range) & $45.1(31-76)$ & $45.4(33-77)$ & 0.59 \\
\hline \multicolumn{4}{|l|}{ Histology [15] } \\
\hline Follicular & $41(67.2)$ & $42(68.8)$ & 0.85 \\
\hline Marginal zone & $17(27.9)$ & $15(24.6)$ & 0.68 \\
\hline Small lymphocytic & $2(3.3)$ & $2(3.3)$ & 1.00 \\
\hline Lymphoplasmocytic $^{\text {a }}$ & $1(1.6)$ & $2(3.3)$ & 0.56 \\
\hline \multicolumn{4}{|l|}{ Stage } \\
\hline II & $3(4.9)$ & $2(3.3)$ & 0.65 \\
\hline III & $12(19.7)$ & $11(18.0)$ & 0.81 \\
\hline IV & $46(75.4)$ & $48(78.7)$ & 0.66 \\
\hline B symptoms & $22(36.1)$ & $19(31.1)$ & 0.56 \\
\hline Bone marrow involved & $40(65.6)$ & $42(68.9)$ & 0.69 \\
\hline Extra-nodal involved sites $\geq 1$ & $46(75.4)$ & $45(73.8)$ & 0.84 \\
\hline $\mathrm{LDH}>240 \mathrm{U} / \mathrm{L}$ & $22(36.1)$ & $21(34.4)$ & 0.85 \\
\hline \multicolumn{4}{|l|}{ Prognostic groups according to FLIPI } \\
\hline Low risk ( $0-1$ risk factor) & $5(12.1)$ & $5(11.9)$ & 0.96 \\
\hline Intermediate risk ( 2 risk factors) & $15(36.6)$ & $13(31.0)$ & 0.59 \\
\hline Poor risk (3-5 risk factors) & $21(51.2)$ & $24(57.1)$ & 0.58 \\
\hline
\end{tabular}

Unless otherwise indicated, data are number of patients, with percentage in parentheses

FLIPI Follicular Lymphoma International Prognostic Index [16]

${ }^{\text {a } W a l d e n s t r o ̈ m ' s ~ m a c r o g l o b u l i n e m i a ~}$
$1220 / \mathrm{mm}^{3}$ (range, 300-1700) for a mean duration of 9 days (range, 7-11).

Overall, 7/61 patients (11.5\%) disrupted chemotherapy schedules due to FN. Of them, three were time-disruptions and four were dose-disruptions (Table 2).

Five patients $(5 / 61,8.1 \%)$ were hospitalized for pneumonia (median days of hospitalization 18, with a range of 6-22) and received intravenous antibiotic treatment with resolution of infectious episodes (Table 3). Extra-hematological toxicity of grade $\geq$ III (bone pain) was observed in six patients $(9.8 \%)$, treated successfully with paracetamol. No patient had to stop the immuno-chemotherapy because of filgrastimrelated side effects.

\section{Findings in the peg-group}

In the peg-group, pegfilgrastim was administered at day 4 for each of the 366 cycles of immuno-chemotherapy performed. The mean of nadir of ANC was $1734 / \mathrm{mm}^{3}$ (range, 880-2110) for a mean duration of 5 days (range, 3-9).
Overall, only $1 / 61$ patients $(1.6 \%)$ disrupted chemotherapy schedules due to FN (time-disruption; Table 2).

Three patients $(3 / 61,4.9 \%)$ were hospitalized for pneumonia (median days of hospitalization 6, range 1-21) and received intravenous antibiotic treatment with resolution of infectious episodes. No patient died during pegfilgrastim. Apart from the advantage of mono-administration, pegfilgrastim was well tolerated in all patients. Extrahematological toxicity of grade III (bone pain) was observed in $7 / 61$ patients $(11.5 \%)$, managed successfully with paracetamol. Extra-hematological toxicity of grade IV (bone pain) was observed only in one patient (1.6\%), who had to stop the treatment.

\section{Peg-group vs. control group}

Thus, pegfilgrastim was significantly associated with fewer incidence rate of FN-related chemotherapy disruptions $(11.4 \%$ in the control group vs. $1.6 \%$ in the peg-group, $p=0.04$ ) and fewer days of hospitalization due to FN (median number 18 days in the control group 
Table 2 Modification of immune-chemotherapy schedule with rituximab plus bendamustine according to Rummel et al. [1] due to neutropenia in the two study groups

\begin{tabular}{llll}
\hline & $\begin{array}{l}\text { Control group } \\
(N=61)\end{array}$ & $\begin{array}{l}\text { Peg-group } \\
(N=61)\end{array}$ & $\begin{array}{l}p \\
\text { value }\end{array}$ \\
\hline Overall chemotherapy disruption & $7(11.5)$ & $1(1.6)$ & 0.028 \\
Time disruption & $3(4.9)$ & $1(1.6)$ & 0.31 \\
$\quad$ Median days (range) & $16(6-21)$ & $5^{\mathrm{a}}$ & 0.04 \\
Dose disruption & $4(6.6)$ & - & 0.04 \\
$\quad$ Percentage & 22.3 & - & $<0.001$ \\
\hline
\end{tabular}

Unless otherwise indicated, data are number of patients, with percentage in parentheses

${ }^{\text {a }}$ Data are from the only patient in the peg-group obliged to delay chemotherapy due to FN vs. 6 in the peg-group, $p=0.04)$. In terms of G-CSFrelated extra-hematological grade III side effects, no significant difference has been found in the two groups (9.8\% in the control group vs. $11.5 \%$ in the peg-group, $p=0.77)$. Only one patient was obliged to stop the treatment in the peg-group due to intolerance.

\section{Discussion}

Immuno-chemotherapy treatment with rituximabbendamustine has been approved as a frontline treatment for indolent NHL. It has been demonstrated to be non-inferior to rituximab in combination with cyclophosphamide,
Table 3 Clinical characteristics of neutropenic episodes in the two study groups

\begin{tabular}{|c|c|c|c|}
\hline Characteristic & $\begin{array}{l}\text { Control group } \\
(N=61)\end{array}$ & $\begin{array}{l}\text { Peg-group } \\
(N=61)\end{array}$ & $\begin{array}{l}p \\
\text { value }\end{array}$ \\
\hline Neutropenic episodes without infectious symptoms & $20(32.8)$ & $7(11.5)$ & 0.04 \\
\hline Febrile neutropenia of unknown origin & $10(16.4)$ & $2(3.3)$ & 0.04 \\
\hline Febrile neutropenia with clinically documented infection & $17(27.8)$ & $5(8.2)$ & 0.005 \\
\hline \multicolumn{4}{|l|}{ Site/source of infection } \\
\hline Mouth $^{\mathrm{a}}$ & $7(11.5)$ & $1(1.6)$ & 0.03 \\
\hline Upper respiratory tract & $3(4.9)$ & $1(1.6)$ & 0.31 \\
\hline Lower respiratory tract & $5(8.2)$ & $3(4.9)$ & 0.46 \\
\hline Urinary tract & 2 & - & \\
\hline Radiological signs of infection ${ }^{\mathrm{b}}$ & $5(8.2)$ & $3(4.9)$ & 0.46 \\
\hline $\begin{array}{l}\text { Febrile neutropenia with microbiologically documented } \\
\text { infections }\end{array}$ & $7(11.5)$ & $4(6.6)$ & 0.34 \\
\hline Bacteremia & $2(3.3)$ & - & 0.15 \\
\hline Gram-positive & $2(3.3)$ & - & 0.15 \\
\hline Enterococcus spp. & $1(1.6)$ & - & 0.31 \\
\hline Staphylococcus spp. & $1(1.6)$ & - & 0.31 \\
\hline Gram-negative & - & - & 1.00 \\
\hline Positive sputum culture & $2(3.3)$ & $1(1.6)$ & 0.56 \\
\hline Gram-positive & $1(1.6)$ & $1(1.6)$ & 1.00 \\
\hline Staphylococcus spp. & $1(1.6)$ & - & 0.31 \\
\hline Gram-negative & $1(1.6)$ & - & 0.31 \\
\hline Pseudomonas spp. & $1(1.6)$ & - & 0.31 \\
\hline Serum CMV DNA positivity & $3(4.9)$ & $3(4.9)$ & 1.00 \\
\hline Hospitalization required for FN complications & $5(8.1)$ & $3(4.9)$ & 0.46 \\
\hline \multicolumn{4}{|l|}{ Hospitalization days } \\
\hline Median (range) & $18(6-22)$ & $6(1-21)$ & 0.04 \\
\hline ICU recovery & $2(3.3)$ & - & 0.15 \\
\hline
\end{tabular}

Unless otherwise indicated, data are number of patients, with percentage in parentheses

${ }^{\text {a } I t ~ r e f e r s ~ t o ~ s t o m a t i t i s ~}$

${ }^{\mathrm{b}}$ Chest radiography or CT scans suspected for pneumonia 
doxorubicin, vincristine, and prednisone (R-CHOP) in terms of efficacy, but better tolerated. Indolent NHL is characterized by a chronic relapsing-remitting disease course, with patients usually exposed to several successive treatment courses.

In this scenario, a reduction of treatment-related toxicities and an improvement of quality of life should be considered so important as clinical results.

In this prospective study, the impact of G-CSF administered during induction treatment with bendamustine plus rituximab for indolent NHL was evaluated by comparing patients who received secondary prophylaxis with filgrastim (control group) vs. patients who received pegfilgrastim as primary prophylaxis (peg-group).

In this study, we observed a lower rate of febrile neutropenia with clinical signs of infection in the peg-group compared with control group ( 8.2 vs. $27.8 \% ; p=0.005$ ): in particular, we observed an increased number of stomatitis in the control group compared with the peg-group ( 11.5 vs. $1.6 \% ; p=0.03$ ).

Conversely, no difference was found between the two groups regarding microbiological documented infection, both for bacterial infection than for those CMV-related. The common prophylaxis to the two study groups with quinolone is likely able to reduce the rate of documented bacterial infections regardless of neutropenia, although the number of patients is probably too low to state this statement with certainty. At the same time, the number of CMV-related infections is equal in the two groups ( 3 vs. 3 ), being this type of viral infection non-controllable merely by neutrophils. Indeed, the number of hospitalization required for FN complications was similar in the two study groups $(8.1 \%$ in the control group vs. $4.9 \%$ in the peg-group), being influenced by the CMVinduced pneumonia; while the median hospitalization time was lower in the peg-group, probably due to a more rapid recovery of neutrophil counts.

Moreover, pegfilgrastim demonstrated a reduction of the incidence rate of FN-related chemotherapy disruptions (regarding dose-dense and/or dose-intensity of schedule), of days of hospitalization due to $\mathrm{FN}$, with no differences in G-CSF-related side effects (grade $\geq 3$ WHO toxicity criteria), such as bone and back pain. No clinical significant changes in laboratory parameters or vital signs were observed.

This gives an advantage in pegfilgrastim's group also in terms of quality of life, which should be always considered in this setting of patients.

In conclusion, in patients affected by newly diagnosed indolent NHL, in treatment with bendamustine plus rituximab, primary prophylaxis with pegfilgrastim seems to reduce the incidence of chemotherapy disruptions due to $\mathrm{FN}$, and of the days of hospitalization, with no differences in G-CSF-related side effects. Moreover, it is well-tolerated and may increase the opportunity to maintain the planned schedule of treatment. These results make pegfilgrastim an advantageous option in most cases both in terms of cost-effectiveness and of quality of life. These preliminary observations need to be validated by controlled clinical trials.

Compliance with ethical standards The protocol was approved by the local ethics committee; the study complied with the Declaration of Helsinki and its amendments. It was done in accordance with the Good Clinical Practice guidelines. All patients gave written informed consent.

Conflict of interest The authors declare that they have no conflicts of interest.

Open Access This article is distributed under the terms of the Creative Commons Attribution-NonCommercial 4.0 International License (http:// creativecommons.org/licenses/by-nc/4.0/), which permits any noncommercial use, distribution, and reproduction in any medium, provided you give appropriate credit to the original author(s) and the source, provide a link to the Creative Commons license, and indicate if changes were made.

\section{References}

1. Rummel MJ, Niederle N, Maschmeyer G et al (2013) Bendamustine plus rituximab versus CHOP plus rituximab as first-line treatment for patients with indolent and mantle-cell lymphomas: an open-label, multicentre, randomised, phase 3 noninferiority trial. Lancet 381(9873):1203-1210. doi:10.1016 /S0140-6736(12)61763-2

2. Luminari S, Goldaniga M, Cesaretti M et al (2016) A phase II study of bendamustine in combination with rituximab as initial treatment for patients with indolent non-follicular non-Hodgkin lymphoma. LeukLymphoma Apr 57(4):880-887

3. Common Terminology Criteria for Adverse Events (CTCAE) (2009) Version 4.0, published: May 28, 2009 (v4.03: June 14, 2010)

4. Link H, Nietsch J, Kerkmann M et al (2016) Adherence to granulocytecolony stimulating factor (G-CSF) guidelines to reduce the incidence of febrile neutropenia after chemotherapy-a representative sample survey in Germany. Support Care Cancer 24(1):367-376

5. Crawford J, Dale DC, Lyman GH (2004) Chemotherapy-induced neutropenia: risks, consequences, and new directions for its management. Cancer 100:228-237

6. Pettengell R, Schwenkglenks M, Leonard R et al (2008) Neutropenia occurrence and predictors of reduced chemotherapy delivery: results from the INC-EU prospective observational European neutropenia study. Support Care Cancer 16:1299-1309

7. Smith TJ, Khatcheressian J, Lyman GH et al (2006) 2006 update of recommendations for the use of white blood cell growth factors: an evidence-based clinical practice guideline. J ClinOncol 24:3187-3205

8. Crawford J, Armitage J, Balducci L, National comprehensive cancer network et al (2013) Myeloid growth factors. J NatlComprCancNetw 11(10):1266-1290

9. Cerchione C, Catalano L, Pareto AE et al (2015) Pegfilgrastim in primary prophylaxis of febrile neutropenia during chemotherapy of relapsed and refractory multiple myeloma: a real-life experience. Support Care Cancer 23(2):301-302

10. Green MD, Koelbl H, Baselga J et al (2003) A randomized double blind multicenter phase III study of fixed-dose single- 
administration pegfilgrastim versus daily filgrastim in patients receiving myelosuppressive chemotherapy. Ann Oncol 14:29-35

11. Balducci L, Al-Halawani H, Charu V et al (2007) Elderly cancer patients receiving chemotherapy benefit from first-cycle pegfilgrastim. Oncologist 12:1416-1424

12. Dreyling M, ESMO Guidelines Working Group (2009) Newly diagnosed and relapsed follicular lymphoma: ESMO clinical recommendations for diagnosis, treatment and follow-up. Ann Oncol 20(Suppl 4):115-118

13. Freifeld GA, Bow EJ, Sepkowitz KA et al (2011) Executive summary: clinical practice guideline for the use of antimicrobial agents in neutropenic patients with cancer: 2010 update by the Infectious Disease Society of America. Clin Infect Dis 52:427-431

14. European Committee on Antimicrobial Susceptibility Testing ( 2014 ) Available at: http://www.eucast. org/fileadmin/src/media/PDFs/EUCAST files/Disk test documents/EUCAST_breakpoints_v1.3_pdf.pdf. Accessed $\overline{3}$ November 2014

15. Zelenetz AD, Gordon LI, Wierda WG et al (2014) Non-Hodgkin's lymphomas, version 4. J Natl Compr Netw 12(9):1282-1303

16. Solal-Céligny P, Roy P, Colombat $P$ et al (2004) Follicular lymphoma international prognostic index. Blood 104(5):1258-1265 